\title{
IRREDUCIBLE SEMIGROUPS
}

\author{
BY K. H. HOFMANN AND P. S. MOSTERT ${ }^{1}$ \\ Communicated by E. Hewitt, February 11, 1964
}

Introduction. Let $S$ be a compact connected semigroup with identity, which we denote by 1 . If $S$ is not a group, then there is a proper unique minimal ideal $M \subset S$ which is itself compact and connected, and hence also there is a minimal compact connected subsemigroup containing the identity and meeting $M$. Such a semigroup we call irreducible. (The property of being irreducible is independent of the semigroup in which such a semigroup is contained.) $)^{2}$ Because every compact connected semigroup with identity which is not a group contains an irreducible semigroup joining its identity to its minimal ideal, the study of these is of utmost importance and a knowledge of their structure appears to be more or less an optimal expectation in the direction of a general structure theorem for compact semigroups (although in particular cases, of course, more can be expected). It is the purpose of this note to announce the structure of several large classes of irreducible semigroups (which in particular includes the abelian ones, a case in which quite a bit is already known from the work of Hunter [5]). The proof is quite lengthy and will appear in our forthcoming book.

The structure of the maximal subgroup of the identity, being a compact group, and of the minimal ideal, being a Rees product of groups [10], is known. Attempts to move out into the remaining portion have been successful in particular cases [2], [3], [7], the degree of success being to a large extent proportional to the success in finding the structure of an irreducible subsemigroup joining the identity and the minimal ideal. In every previous case in which this structure has been found, the irreducible semigroup has been an $I$-semigroup (i.e., a semigroup with identity on an arc such that the minimal ideal reduces to one end point [7]) or the closure of a one-parameter subsemigroup [1]. However, examples of much more complicated irreducible semigroups have been given by Hunter [4], and some information about these semigroups was obtained by him and Rothman [6] and by Rothman [8], [9], mainly in the case of normal semi-

\footnotetext{
1 This work was partially supported by NSF Grant GP1877.

${ }^{2}$ An equivalent definition is: a semigroup is irreducible if it is compact, connected, has an identity, and contains no proper compact connected subsemigroup containing its identity and meeting its minimal ideal. In the terminology of Hunter and Rothman, this is a semigroup algebraically irreducible between the identity and some idempotent in the minimal ideal.
} 
groups (i.e., semigroups $S$ such that $x S=S x$ for every $x$ ), and in particular by Hunter [5], where the abelian case was almost characterized, but the full structure was left unsettled. (It will be clear that all previous results in this direction can be derived quite simply from our structure theorem.)

1. The hormos. In this section we describe a process by which a large class of semigroups may be constructed. ${ }^{3} \mathrm{~A}$ more general process will appear in our book, but this will suffice to describe the classes of irreducible semigroups we shall consider. The intuitive notion involves a chaining process in which compact semigroups of a particularly nice nature are chained together in a similar fashion to that of chaining interval semigroups to form the class of $I$-semigroups [7]. For this reason, we call this construction a hormos, from opmos, meaning "ornamental chain." In the construction, it will be helpful to keep in mind the examples of Hunter and Rothman [6], even though these examples do not reflect the full nature of the construction in the most general situation.

The construction. The hormos will be constructed from a collection of objects $\left(X, S_{x}, m_{x y}\right)$ where

(a) $X$ is a totally ordered compact set with minimal element 0 and maximal element $m$. We shall denote by $X^{\prime}$ the set of all elements $x>0$ such that $] y, x[=\varnothing$ for some $y<x$, where $] y, x[=\{z \in X$ : $y<z<x\}$. (Of course $X$ is connected if and only if $X^{\prime}$ is empty.)

(b) $S_{x}$, for each $x \in X$, is a compact semigroup with identity $1_{x}$. We let $G_{x}$ denote the maximal subgroup for which $1_{x}$ is the idempotent, and $M_{x}$ the minimal ideal of $S_{x}$. The following conditions on these objects are assumed satisfied:

(i) $M_{x}$ is a group with idempotent $e_{x}$ (this implies $s e_{x}=e_{x} s$ for all $\left.s \in S_{x}\right)$.

(ii) If $x \notin X^{\prime}$, then $S_{x}=G_{x}=M_{x}$.

(c) $m_{x y}: S_{y} \rightarrow G_{x}$ is a homomorphism for each $x<y$ such that

$$
\begin{aligned}
& m_{x y} m_{y z}=m_{x z} \quad \text { for } \quad x<y<z, \\
& m_{x y}(s)=m_{x y}\left(s e_{y}\right)=m_{x y}\left(e_{y} s\right), \quad s \in S_{y} .
\end{aligned}
$$

(We shall denote the identity function on $S_{x}$ by $m_{x x}$.)

(d) $m_{x y} \mid M_{y}$ is an injection if $] x, y[=\varnothing$.

(e) The function $\phi_{x}: G_{x} \rightarrow \prod\left\{G_{z}: z<x\right\}$ defined by

3 This construction is, up to a point and from the purely algebraic point of view, quite similar to that used by Clifford (Semigroups admitting relative inverses, Ann. of Math. (2) 42 (1947), 1037-1049) in the study of semigroups which are unions of groups. However, we extend this both algebraically and topologically. 


$$
\phi_{x}(g)_{z}=m_{z x}(g)
$$

for $0<x \notin X^{\prime}$, where $\prod\left\{G_{z}: z<x\right\}$ is given the product topology, is an isomorphism onto the projective limit

$$
\lim _{\leftarrow}\left\{G_{z}, m_{z y}, z<y<x\right\} \subset \prod\left\{G_{z}: z<x\right\} .
$$

With these building blocks, we now proceed with the construction of a new semigroup as follows:

Let $P$ denote a disjoint union of the semigroups $S_{x}$, and $p: P \rightarrow X$ the function which is defined by $p(s)=x$ if $s \in S_{x}$. We define multiplication in $P$ by

$$
s \cdot t=m_{\mu p(s)}(s) \cdot m_{\mu p(t)}(t), \quad \mu=\min \{p(s), p(t)\} .
$$

We now define

$$
\left.\left.m_{x}: p^{-1}(] x, m\right]\right) \rightarrow G_{x}
$$

by

$$
m_{x}(s)=m_{x p(s)}(s),
$$

and by means of these functions a basis $B$ for a topology on $P$ by

(a) any open set of $S_{x}-G_{x}$ is in $B$.

(b) If $W$ is an open interval in $X, w=$ g.1.b. $W$, and $U$ is any open set in $S_{w}$, then

$$
V(W, U)=p^{-1}(W) \cap\left(U \cup m_{w}^{-1}\left(U \cap G_{w}\right)\right)
$$

is in $B$.

With this topology, $P$ becomes a compact semigroup. Now we define the following relation $R$ on $P$ : The cosets of $R$ are $\left\{m_{\mu \nu}(s), s\right\}$ if $] \mu, \nu\left[=\varnothing, p(s)=\nu\right.$ and $s \in M_{\nu}$ and are singleton sets otherwise.

It follows that $R$ is a closed congruence relation so that the space $T=P / R$ is a compact semigroup. Since there is a natural embedding of $S_{x}$ in $T$, we shall identify $S_{x}$ with its image under the embedding. Then $1_{m}$ is the identity for $T$ and $G_{0}$ is the minimal ideal. This semigroup $T$ we denote by

$$
T=\operatorname{Horm}\left(X, S_{x}, m_{x y}\right)
$$

and call it the hormos over the system $\left(X, S_{x}, m_{x y}\right)$.

2. Completely irreducible semigroups. In this paragraph we describe the structure of the "building blocks" which are used in the construction of an irreducible hormos. 
An irreducible semigroup is said to be completely irreducible if it contains no proper irreducible semigroup outside the minimal ideal. Equivalently, an irreducible semigroup is completely irreducible if any idempotent which is not the identity lies in the minimal ideal. We shall say that a semigroup $S$ is irreducible with respect to its maximal subgroup $G$ if $S$ is a connected compact semigroup with identity which contains no proper connected compact subsemigroup containing $G$ and meeting the minimal ideal. It is then completely irreducible with respect to its maximal subgroup if for any connected compact subsemigroup $S^{\prime}$ with identity such that $G S^{\prime} G \subset S^{\prime}$, it follows that $S^{\prime}=S$, or $S^{\prime} \subset G$ or $S^{\prime}$ is contained in the minimal ideal.

In the characterization of irreducible semigroups, one is able to build up, using techniques of Koch, Mostert and Shields, chains of subsemigroups each of which is a hormos with abelian maximal group. In order to apply a tower argument and not to build up undesired structure at the same time, it is necessary to generalize these procedures in order to find the subsemigroups in the centralizer of the maximal subgroup of an idempotent, or at least of any abelian subgroup thereof. Hence a complete characterization for irreducible semigroups demands first the characterization of semigroups which are completely irreducible with respect to their maximal subgroups, and indeed this may be the most difficult remaining step, although one must also treat the case where $S$ has an $I$-semigroup of idempotents whose maximal element is the identity. The problem in both cases is to show that the component of 1 of the centralizer of the maximal subgroup $G$ of the identity contains points not in $G$. For normal semigroups, and for certain other classes, we are able to obtain this. We now describe a class of semigroups which are completely irreducible with respect to their maximal subgroups. It is our belief that these are all of them, but we are not able to prove this at this time.

Let $A$ be a compact connected abelian group with a dense oneparameter subgroup and $f:[0, \infty[\rightarrow A$ a monomorphism of the additive semigroup of non-negative real numbers with dense image. Addition on $[0, \infty[$ can be extended continuously to the one point compactification $[0, \infty]$ so that $a+\infty=\infty=\infty+a$ for all $a \in[0, \infty]$ (and in fact is isomorphic under the natural extension of $x \rightarrow \exp x$ with the unit interval $[0,1]$ under multiplication.) The space $[0, \infty]$ $\times A$ is a compact semigroup with respect to coordinatewise multiplication. Define the subsemigroup

$$
\Sigma(f, A, \infty)=\{(t, f(t)): t \in[0, \infty)\} \cup\{\infty\} \times A .
$$


Let $0 \leqq a \leqq \infty$, and let $Q$ be the congruence relation defined by $\left((t, h),\left(t^{\prime}, h^{\prime}\right)\right) \in Q$ if $h=h^{\prime}$ and either $t=t^{\prime}$, or $t, t^{\prime} \geqq a$. Define $\Sigma(f, A, a)$ $=\Sigma(f, A, \infty) / Q$. Then $\Sigma(f, A, a)$ is a completely irreducible semigroup.

Now let $G$ be any compact group, $\mathfrak{R}$ a collection of closed normal subgroups of $A \times G$, and $F:[0, \infty] \rightarrow \mathfrak{N}$ a function satisfying

(i) $F(0)=\{(1,1)\}$,

(ii) $F(a) \cap A \times\{1\}=\{(1,1)\}$,

(iii) $s<t$ implies $F(s) \subset F(t)$,

(iv) $a<t$ implies $F(a)=F(t)$,

(v) $\cap\{F(t): t>s\}=F(s), s<a$,

(vi) $A \times B / F(a)$ is connected.

Then $F$ determines a closed congruence relation on $\Sigma(f, A, a) \times G$, which we again denote by $F$, by

$$
\left((t, h, g),\left(t^{\prime}, h^{\prime}, g^{\prime}\right)\right) \in F \text { if } t=t^{\prime} \text { and }\left(h^{-1} h^{\prime}, g^{-1} g^{\prime}\right) \in F(t) .
$$

Then $(\Sigma(f, A, a) \times G) / F$ is completely irreducible with respect to the maximal subgroup $\{(0,1)\} \times G$. We have then the following results, of which the first is known. (See [1] for example.)

THEOREM A. A compact semigroup is completely irreducible if and only if it is isomorphic to some $\Sigma(f, A, a)$.

It is conjectured that a semigroup $S$ which is completely irreducible with respect to its maximal subgroup can contain no idempotent other than the identity outside its minimal ideal.

We list here nine properties which a semigroup $S$ may have, any one of which will enable us to characterize the irreducible semigroups having that property. In the case of affine semigroups, this is of course trivial since every idempotent $f$ determines a semigroup $\{(1-\alpha) \cdot 1$ $+\alpha \cdot f: 0 \leqq \alpha \leqq 1\}$ isomorphic to $\Sigma(f,\{1\}, \infty)$ However, we desire even more, and that is that such an irreducible semigroup exist in the centralizer of the maximal subgroup, and this is not trivial in any of these cases except for idempotent irreducible semigroups satisfying P1.

P0. Each component of the set of idempotents is an abelian set.

P1. $S x=x S$ for all $x \in S$.

P2. For each idempotent $e$ not in the minimal ideal, and $x \in e S e$, $x H_{e} \subset H_{e} x$ or $x H_{e} \supset H_{e} x$ where $H_{e}$ is the maximal subgroup with $e$ as identity.

P3. Some neighborhood of $H_{e}$ in $e S e$ has an embedding under a local isomorphism into a Lie group for every idempotent $e$ not in the minimal ideal where $H_{e}$ is the maximal subgroup with $e$ as identity. 
P4. The $D$-class decomposition of $S$ is an arc and the set of idempotents is totally ordered under $e \leqq f$ if $e \in f S f$.

P5. The $L$-class and $R$-class decompositions are totally ordered.

P6. $S$ is an affine semigroup.

P7. $S$ is isomorphic to a semigroup of matrices.

REMARK. In an irreducible matrix semigroup, there can be at most $n+1$ idempotents where $n$ is the rank of the identity matrix of the semigroup.

PK. The $D$-class of every idempotent is the same as its $H$-class (which is the maximal group of this idempotent).

REMARK. This condition has been suggested by R. J. Koch.

TheOREM B. A compact semigroup which satisfies one of the properties $\mathrm{P} 1, \cdots, \mathrm{P} 7, \mathrm{PK}$ is completely irreducible with respect to its maximal subgroup if and only if it is isomorphic to $(\Sigma(f, A, a) \times G) / F$ for some collection $(f, A, a, G, F)$. If the maximal group is abelian then this conclusion holds also if $\mathrm{P} 0$ is satisfied; then $S$ is abelian.

Theorem C. Let $S$ be a hormos. Then $S$ is irreducible if and only if $S=$ Horm $\left(X, S_{x}, m_{x y}\right)$, where

(i) $S$ is abelian.

(ii) $S_{x}=\left(\Sigma\left(f_{x}, A_{x}, a_{x}\right) \times G_{x}\right) / F_{x}$ for some system $\left(f_{x}, A_{x}, a_{x}, G_{x}, F_{x}\right)$.

(iii) $G_{m}=\{1\}$.

(iv) $\left.\left.f_{x}: p^{-1}\right] x, m\right] \rightarrow G_{x}$ is surjective for every $x \in X$.

TheOREM D. Let $S$ be a compact connected semigroup with identity which satisfies one of $\mathrm{P} 0, \mathrm{P} 1, \cdots, \mathrm{P} 7, \mathrm{PK}$. Then $S$ is irreducible if and only if it is isomorphic to an irreducible hormos.

ThEOREM E. Let $S$ be a compact connected semigroup with identity satisfying one of $\mathrm{P} 1, \cdots, \mathrm{P} 7$. Then $S$ contains an irreducible hormos $T$ which contains the identity of $S$, meets the minimal ideal, and is contained in the centralizer of the maximal subgroup of the identity. In the case of P0 and PK, an irreducible hormos containing the identity and meeting the minimal ideal can be found in the centralizer of any compact connected abelian subgroup of the maximal subgroup.

\section{BIBLIOGRAPHY}

1. K. H. Hofmann, Topologische Halbgruppen mit dichter submonogener Unterhalbgruppen, Math. Z. 74 (1960), 232-276.

2. - Locally compact semigroups in which a subgroup with compact complement is dense; Homogeneous locally compact groups with compact boundary, Trans. Amer. Math. Soc. 106 (1963), 19-51; ibid. 106 (1963), 52-63.

3. A. L. Hudson, Some semigroups on an n-cell, Trans. Amer. Math. Soc. 99 (1961), 255-263. 
4. R. P. Hunter, Note on arcs in semigroups, Fund. Math. 49 (1961), 233-245.

5. - On the structure of homogroups with applications to the theory of compact connected semigroups, Fund. Math. 52 (1963), 69-102.

6. R. P. Hunter and N. J. Rothman, Characters and cross sections, Duke Math. J. 29 (1962), 347-366.

7. P. S. Mostert and A. L. Shields, On the structure of semigroups on a compact manifold with boundary, Ann. of Math. (2) 65 (1957), 117-143.

8. N. J. Rothman, On algebraically irreducible semigroups, Duke Math. J. (to appear).

9. - Linearly quasi-ordered compact semigroups, Proc. Amer. Math. Soc. 13 (1962), 352-357.

10. A. D. Wallace, The Rees-Suchkewitsch structure theorem for compact simple semigroups, Proc. Nat. Acad. Sci. U.S.A. 42 (1956), 430-432.

TUlane UNIVERSITY OF LOUISIANA 